\title{
Determining Atomic Structures from Digitally Defined Regions of Nanocrystals
}

Marcus Gallagher-Jones ${ }^{1}$, Karen Bustillo ${ }^{2}$, Colin Ophus ${ }^{3}$, Logan Richards ${ }^{1}$, Jim Ciston ${ }^{3}$, Sangho Lee ${ }^{4}$, Andrew Minor $3^{3,5}$ and Jose Rodriguez ${ }^{1}$

${ }^{1}$ University of California-Los Angeles, Los Angeles, California, United States, ${ }^{2}$ NCEM, Molecular Foundry, LBNL, Berkeley, California, United States, ${ }^{3}$ Lawrence Berkeley National Laboratory, Berkeley, California, United States, ${ }^{5}$ Sungkyunkwan University, Seoul, Seoul-t'ukpyolsi, Republic of Korea, ${ }^{6}$ University of California-Berkeley, Berkeley, California, United States

The ability of molecules to form ordered assemblies is a crucial first step in preparing samples for structural characterization with atomic-level detail. For many complex molecules the length scales to which this order extends is limited, thus hampering efforts to solve their structures. Nanocrystallography has facilitated the characterization of many new molecular structures by reducing the required sizes of crystals being interrogated to tens to hundreds of nanometres [1-3]. 4-dimensional scanning transmission electron microscopy (4D-STEM), a scanning diffraction technique, provides a means to measure long- to medium-range order in crystalline assemblies with single nanometer spatial resolution [4]. 4D-STEM, in conjunction with cryogenic techniques, has recently been applied to investigate crystalline order in a variety of radiation-sensitive organic polymers [5-7]. In our current work we extend these developments in 4D-STEM by incorporating tomography to solve atomic structures of macromolecules from specific regions of polymer nanocrystals.

In this method, scanning nanobeam electron diffraction tomography (nanoEDT), peptide crystals $<0.5$ $\mu \mathrm{m}$ in thickness/width and several $\mu \mathrm{ms}$ in length are rotated about a tilt axis in one-degree steps. At each tilt angle a 4D-STEM dataset is collected by scanning a $12 \mathrm{~nm}$ electron beam in $40 \mathrm{~nm}$ steps across the sample; a diffraction pattern is acquired from each of the scanning beam probe positions (Fig. 1(a)). A direct electron detector captures thousands of sparse diffraction patterns mapped to specific locations within a single crystal over multiple crystal orientations (Fig.1(c)). The use of direct electron detection, in combination with data collection at cryogenic temperatures and a hybrid counting algorithm, allows even weak signals from high-resolution Bragg peaks to be accurately recorded from radiation sensitive crystals (Fig. 1(d)). This intensity data, representing an angular wedge of reciprocal space (Fig. 1(e)), is extracted and used to compute the structure of an amyloid-forming segment from the OsPYL/RCAR5 protein at atomic resolution using fragment-based phasing methods (Fig. 1(f)).

By scanning the beam, we collect diffraction from a wide field-of-view and digitally recombine it for later analysis, obviating the need for a selected-area aperture. Data collected by nanoEDT compares favorably with data acquired under more conventional electron diffraction methodologies, with minimal evidence of radiation damage (Fig. 2). NanoEDT breaks new ground in nanocrystallography by allowing atomic structures to be determined from any region of a nanocrystal through the use of virtual, selected-area apertures, potentially leading to the determination of atomic structures from heterogeneous or polycrystalline nanoassemblies [8]. 


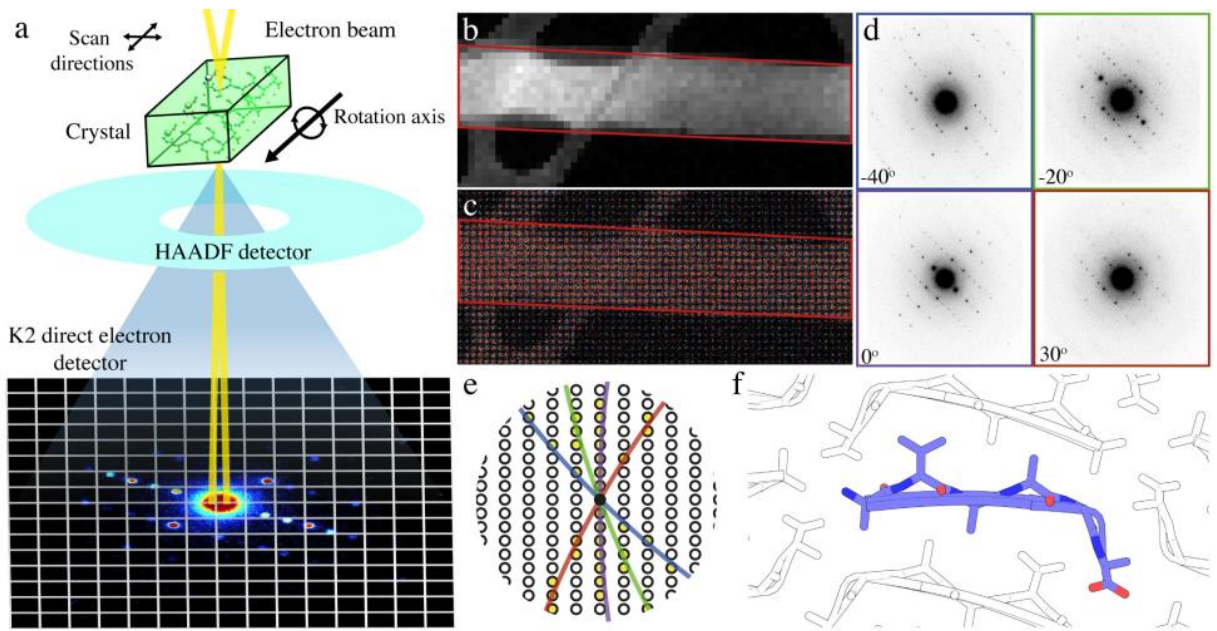

Figure 1. (a) Schematic of the experimental setup for nanoEDT with key components highlighted. (b) Annular dark-field image of a peptide nanocrystal. (c) Composite image of all diffraction patterns collected simultaneously with (b) during a 4DSTEM scan. (d) Examples of diffraction patterns computed from data collected at discrete orientations during nanoEDT. (e) Assembly of the intensities extracted from (d) into a reciprocal lattice. (f) Atomic structure of OsPYL/RCAR5 peptide solved by nanoEDT.

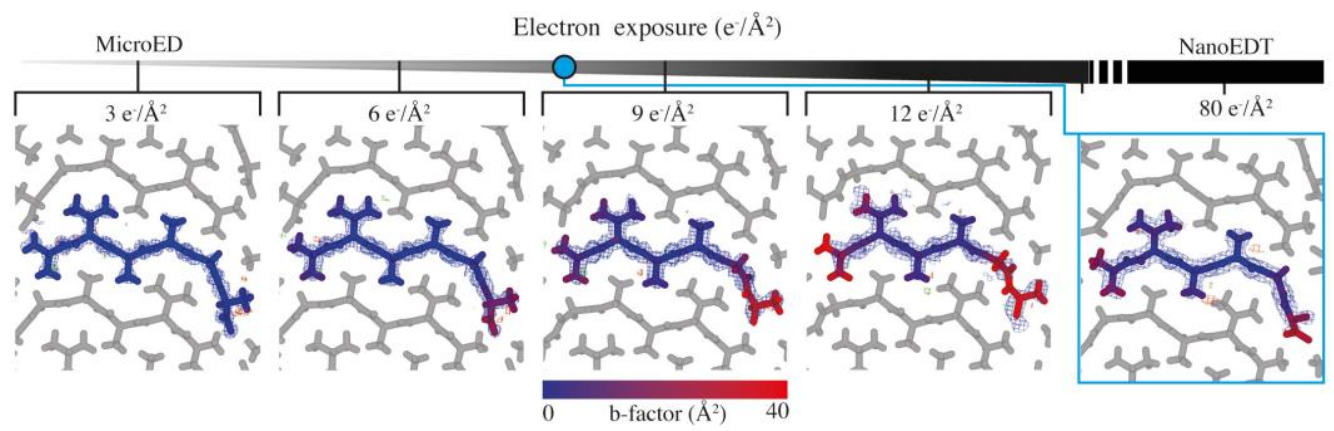

Figure 2. Comparison of atomic displacements in structures solved by electron diffraction at different electron exposures. While the estimated exposure of nanoEDT is high, the observed displacements are more consistent with a lower exposure being experienced by the crystal during the experiment (blue circle).

\section{References}

[1] A. Lanza et al. IUCrJ 6, (2019).

[2] E. Mugnaioli et al. Inorganic chemistry 57 (2018), p. 10241-10248.

[3] M. R. Sawaya et al. PNAS 113 (2016), p. 11232-11236.

[4] C. Ophus Microscopy and Microanalysis (2019), p. 1-20.

[5] O. Panova et al. Micron 88 (2016), p. 30-36.

[6] O. Panova et al. Nature Materials 18 (2019), p. 860 - 865

[7] M. Gallagher-Jones et al. Communications Biology 2 (2019), 26.

[8] This work was supported by STROBE, a Science and Technology Center sponsored by the National Science Foundation. Work at the Molecular Foundry was supported by the Office of Science, Office of Basic Energy Sciences, of the U.S. Department of Energy under Contract No. DE-AC02-05CH11231. JC acknowledges additional support from the Presidential Early Career Award for Scientists and Engineers (PECASE) through the U.S. Department of Energy. J.A.R. is supported as a Searle Scholar, a Pew Scholar, a Beckman Young Investigator and a Packard Fellow. 